\title{
The rise of targeted HIV oral rapid testing in Australia
}

\begin{abstract}
TGA approval
of this test

might slow

increasing HIV

infection rates

among MSM

and others

by facilitating
\end{abstract}

diagnosis and

treatment

Derek Chan
MPH, FAChSHM, PhD

Michael Stewart BIntS, MIPH

Maggie Smith

RN, CertSexual ReproductiveHealth

Tony Price BN, MNurs(Ed)

Jo Lusk MBChB, FAChSHM, MPH ${ }^{3}$

Catriona Ooi MBBS, BSc(Med), FAChSHM ${ }^{4}$

Phillip Read MRCP, MPH, FAChSHM ${ }^{5}$

Robert Finlayson

MBBS, FAChSHM ${ }^{6}$

1 Albion Centre, South Eastern Sydney Local Health District.

Sydney, NSW.

2 University of New South Wales,

Sydney, NSW.

3 Short Street Clinic

Sydney, NSW.

4 Western Sydney

Sexual Health Centre

Sydney, NSW.

5 Kirketon Road Centre,

Sydney, NSW.

6 Taylor Square Private Clinic,

Sydney, NSW.

derek.chan2@sesiahs.

health.nsw.gov.au

doi: 10.5694/mjal4.01292

Online first 9/03/15
G eneral awareness of HIV in Australia has declined since the mid 1980s when the "Grim Reaper" campaign depicted the threat of AIDS to everyday Australians. This is due largely to antiretroviral therapy, which has improved quality of life and reduced mortality among people with AIDS; but the incidence of HIV in Australia has steadily increased since the nadir of 724 infections in 1999, to a 15 -year high of 1236 cases in 2013. ${ }^{1}$ This increase has happened despite $90 \%$ uptake of antiretroviral therapy among patients with $\mathrm{HIV},{ }^{1}$ more of them attaining undetectable viral load (58\% in 2004 compared with $88 \%$ in 2013$)^{1}$ and ongoing prevention efforts (safer sex promotion, testing campaigns, post-exposure prophylaxis).

Men who have sex with men (MSM) continue to account for most diagnoses $(85 \%)$ of newly acquired HIV infection (ie, acquired in the past 12 months). ${ }^{1}$ A multidimensional approach - including promotion of safer behaviour, increased HIV testing, early diagnosis, antiretroviral therapy and biomedical prevention - is called for. Australia's Seventh national HIV strategy 2014-2017 aims to reduce the national incidence of HIV infection by $50 \%$ by $2015 .^{2}$

In New South Wales, data from the first three quarters of 2014 showed 263 new HIV diagnoses: 211 (80\%) were in MSM, 37 (14\%) were heterosexually acquired, five $(2 \%)$ came from injecting drugs and nine $(3 \%)$ were from unknown sources of exposure. ${ }^{3}$ The proportion of new infections among MSM increased from $71 \%$ in 2009 to $84 \%$ in 2011, thereafter plateauing at about $80 \%$ per year. ${ }^{3}$ Despite this increase, the overall incidence of HIV infections in NSW has remained relatively stable at 5.3 per 100000 population between 2004 and 2013. ${ }^{1}$

The NSW HIV strategy 2012-2015 aims to reduce HIV transmission among MSM by $60 \%$ by 2015 and $80 \%$

\section{Abstract}

Objective: To assess the performance and acceptability of the OraQuick Advance Rapid HIV-1/2 Antibody Test (ORT) in Australia.

Design, participants and setting: Cross-sectional study of 1074 men who have sex with men (MSM) and individuals aged 18 years or older at high risk of acquiring HIV infection who attended five public HIV or sexual health services, two general practices and one community clinic in Sydney from 1 January to 31 December 2013.

Intervention: One ORT confirmed by fourth-generation HIV enzyme immunoassay (EIA).

Main outcome measures: ORT sensitivity and specificity compared with EIA; acceptabiity of the ORT to participants.

Results: $83.5 \%$ of participants were MSM, $90.3 \%$ were aged under 50 years, and 9\% had never been tested for HIV. There were 11 true-positive ORT results, two false-negative (non-reactive) results (both were early infections), and one false-positive (reactive) result (due to reader error). Sensitivity and specificity were $84.6 \%$ and $99.8 \%$, respectively (compared with a sensitivity of $99.3 \%$ and specificity of $99.8 \%$ listed by the manufacturer). Three quarters of participants (74.0\%; 730/987) found the ORT less stressful than venous sampling. Those who usually had tests at intervals of greater than 3 months deemed the ORT less stressful than those who had quarterly tests $(77.5 \%$ v $64.8 \% ; P<0.001)$. Nearly all participants (99.2\%; 998/1006) would have an ORT again and $99.4 \%$ (994/1000) would recommend it to peers. Most participants (69.1\%; 720/1042) felt ORT approval by Australia's Therapeutic Goods Administration (TGA) would encourage testing.

Conclusion: ORT sensitivity is reduced in early HIV infection. The test is highly acceptable and less stressful than venous sampling. Participants are keen to be tested with the ORT in future, would recommend it to peers and would have tests more frequently if the ORT were licensed. TGA approval of this test might slow increasing HIV infection rates among MSM and others by facilitating diagnosis and treatment.

by $2020 .{ }^{4}$ Increasing the amount of testing is a cornerstone of this strategy; but individuals may elect not to test. Reasons for this include perceiving themselves to be at low risk, fear of a positive result, stress associated with waiting for a result, general reluctance to attend health services, reluctance to re-attend health services to receive results (positive or negative) and fear of venepuncture or generalised discomfort with needles. ${ }^{5,6}$ The stigma of having HIV itself is another reason. ${ }^{7}$

Such barriers to being tested may be overcome by the use of HIV rapid tests that are reliable (sensitive and specific), efficient (render results quickly) and acceptable to the individuals being tested. However, it is important to remember that rapid tests are screening tests and do not replace diagnostic serological tests.

At the time of writing, the Alere Determine HIV 1/2 Ag/Ab Combo test (Alere Pty Ltd), requiring a fingerprick blood sample, was the only HIV rapid test approved by Australia's Therapeutic Goods Administration (TGA). The manufacturer lists the sensitivity and specificity of this test as $100 \%$ and $99.66 \%$, respectively. ${ }^{8}$

The OraQuick Advance Rapid HIV1/2 Antibody Test (ORT) (OraSure Technologies Inc), requiring an oral fluid sample, is currently being considered by the TGA for approval. The ORT was approved by the United States Food and Drug Administration (FDA) for clinical use in 2004 and for home testing in 2012. Serological testing is recommended to confirm 
1 Standard results of the OraQuick Advance Rapid HIV-1/2 Antibody Test
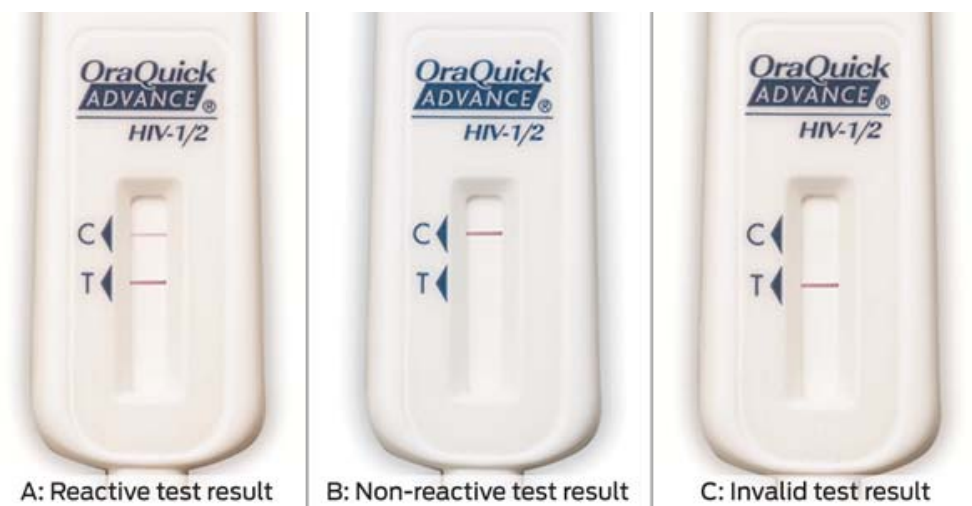

C 4 indicates the control line and T 4 indicates the HIV-1/2 antibody test line. The control line must be present for the test to be valid. Lines at both $\mathrm{C} \mathbf{\uparrow}$ and $\mathrm{T} \mathbf{}$ indicate a reactive test; lines may vary in intensity but must fall in the centre of each $\mathbf{\uparrow}$. Reactive test results should be confirmed with a standard HIV test such as enzyme immunoassay. Tests with invalid results should always be repeated.

Reproduced with permission from OraSure Technologies Inc and Integrated Sciences Pty Ltd.

reactive results of this test. The manufacturer lists the sensitivity and specificity of the ORT as $99.3 \%$ and $99.8 \%$, respectively. ${ }^{9}$

Here, we report the first large-scale Australian study assessing the performance of the ORT compared with standard serological testing (enzyme immunoassay; EIA) in the clinical setting, and its acceptability to individuals at high risk of HIV infection.

\section{Methods}

Between 1 January and 31 December 2013, we conducted a cross-sectional study in eight NSW sites with high MSM caseloads (five public HIV or sexual health services, two general practices and one community clinic). Eligible participants were aged 18 years or older, consented to testing with one ORT plus a confirmatory fourth-generation EIA (either an Elecsys HIV Combi PT [Roche Diagnostics] or Architect HIV Ag/Ab Combo [Abbott Diagnostics] assay, depending on the local laboratory).

\section{Confirmatory tests}

Reactive ORT results were confirmed with the Serodia HIV 1/2 particle agglutination assay (Fujirebio Inc), Genscreen HIV 1/2 Antibody EIA (Bio-Rad Laboratories), Genscreen HIV-1 Ag Assay immunoassay (for p24 antigen; Bio-Rad Laboratories) and the HIV Blot 2.2 Western Blot Assay (MP Diagnostics). Test kits were funded by the South Eastern Sydney Local Health District HIV and Related Programs Unit. Quality assurance was performed externally by the National Reference Laboratory (Point of Care Test External Quality Assessment Scheme Pilot 2013), and internally with manufacturer-supplied control solutions.

\section{Testing protocol}

Before being tested, participants were surveyed for risk history, demographics and their "usual" frequency of HIV testing. Site investigators or trained staff conducted one ORT as per the manufacturer's instructions. The two-step process involved sweeping the test swab across participants' upper and lower gum lines and submerging it in a developer solution vial; this yielded an HIV-1/2 antibody result in 20 minutes (minimum).

Each ORT was interpreted by two staff members and results were reported as reactive, non-reactive or invalid (Box 1). A second ORT was performed for invalid tests (invalid in this context is not synonymous with "indeterminate", as in evolving western blots). After the test, participants with non-reactive results were surveyed about how stressful they found the testing process and whether they would have an ORT in the future or recommend this test to their peers. They were not asked whether having to pay for the test would affect their desire to be tested again, recommend the test or be tested more frequently. Participants with reactive test results were not surveyed and received immediate counselling and expedited medical review. All ORT results were confirmed by the study site laboratory using the available EIA, with results available in 1 to 5 days. Data were analysed using SPSS version 20 (SPSS Inc).

\section{Ethics approval}

The South Eastern Sydney Local Health District Human Research Ethics Committee approved this study (Study 12/139).

\section{Results}

Overall, 1074 participants were tested; $83.5 \%$ (874/1046, 28 null responses) were MSM, and $90.3 \%$ were aged less than 50 years (969/1073, 1 null response). The self-reported frequency of HIV testing for study participants is shown in Box 2.

Box 3 shows that, overall, there were 11 true-positive results (10 MSM and one heterosexual man - all had been tested before), two false non-reactive results (early infections - nonreactive on ORT, negative result on EIA HIV-1/2 antibody test, positive for p24 antigen, indeterminate on

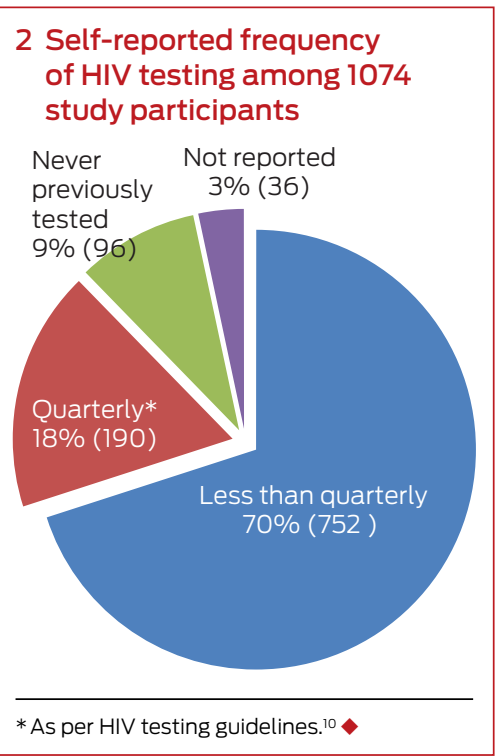


western blot) and one false-positive result (due to reader error). The sensitivity and specificity of the test were $84.6 \%$ and $99.8 \%$, respectively. Results did not differ by age, sexual orientation, number of sexual partners, or testing frequency.

Not all participants answered all questions about ORT acceptability. Of those who completed the survey:

- $74.0 \%$ (730/987) found the ORT less stressful than standard venous sampling (EIA);

- those who usually had tests less frequently (at intervals of greater than 3 months) deemed the ORT less stressful than those who had more frequent (quarterly) tests $\left(77.5 \%\right.$ v $64.8 \% ; \chi^{2}=16.217 ; \mathrm{df}=1$; $P<0.001)$;

- $99.2 \%$ (998/1006) would have another ORT;

- $99.4 \%$ (994/1000) would recommend the ORT to peers; and

- $69.1 \%$ (720/1042) felt TGA approval would encourage them to test more.

\section{Discussion}

We consider our study population as broadly representative of urban MSM and individuals at high risk of acquiring HIV infection in Australia. The ORT is a potential alternative HIV rapid test to the Alere Determine HIV $1 / 2 \mathrm{Ag} / \mathrm{Ab}$ Combo test. As stated earlier, both tests have high (and similar) sensitivity and specificity under clinical trial conditions. ${ }^{8,9}$ However, in actual clinical use, they perform less well. In our study, ORT sensitivity and specificity were $84.6 \%$ and $99.8 \%$, respectively. This compares to a clinical trial of 3190 Alere Determine tests and parallel serological tests in MSM who were offered one or more tests in Sydney, in which the sensitivity and specificity were $87.2 \%$ (94.4\% for antibody, $0 \%$ for antigen, contributing $33 \%$ of false-positive results) and $99.4 \%$, respectively. ${ }^{11}$ Overall, both tests perform similarly under clinical trial conditions and in clinical use, and both are portable and yield results in about 20 minutes.

The main advantage of the ORT is the simple two-step process, making

$$
\begin{aligned}
& 3 \text { Results of the OraQuick Advance Rapid HIV-1/2 Antibody Test for the } 1074 \text { study } \\
& \text { participants }
\end{aligned}
$$

\begin{tabular}{lccccc} 
& & \multicolumn{4}{c}{ Result } \\
\cline { 3 - 6 } Site & $\begin{array}{c}\text { Total no. } \\
\text { of tests }\end{array}$ & $\begin{array}{c}\text { True- } \\
\text { positive }\end{array}$ & $\begin{array}{c}\text { False- } \\
\text { positive }\end{array}$ & $\begin{array}{c}\text { True- } \\
\text { negative }\end{array}$ & $\begin{array}{c}\text { False- } \\
\text { negative }\end{array}$ \\
\hline Public HIV or sexual health service $(n=5)$ & 871 & 9 & 1 & 859 & 2 \\
General practice $(n=2)$ & 129 & 2 & 0 & 127 & 0 \\
Community clinic $(n=1)$ & 74 & 0 & 0 & 74 & 0 \\
Total & 1074 & 11 & 1 & 1060 & 2 \\
\hline
\end{tabular}

it ideal for home self-testing. In contrast, the Alere Determine test requires a blood spot by finger-prick, an exact aliquot to be pipetted onto the test strip and a buffer solution to be applied - a process that is less ideal for home self-testing.

The ORT does, however, have a potential limitation in that its sensitivity may be reduced in two contexts.

Reader error: In a study submitted to the US FDA Blood Products Advisory Committee before its approval of the home-testing kit in 2012, 5499 untrained individuals collected their own samples and interpreted their test results at home as if they had purchased the kit over the counter, ${ }^{12}$ revealing a test sensitivity of $92 \%$ (compared with the ORT sensitivity of $99.3 \%$ listed by the manufacturer ${ }^{9}$ ) while the specificity was $99.8 \%$ (the same as listed for the ORT by the manufacturer ${ }^{9}$ ). The low sensitivity shown in that study may cause confusion and requires explanation. A sensitivity analysis of 114 participants was conducted, of whom 106 returned true-positive results (ie, ORT reactive, positive confirmatory serological test result), but eight incorrectly reported non-reactive results when the serological test result was positive. Thus, the sensitivity was $92.0 \%(106 / 114$; 95\% CI, 86.6\%-96.9\%). ${ }^{12}$ As reader error can lower ORT sensitivity, it is important to confirm negative results with serological tests, especially if seroconversion or early infection is suspected. Note that the sensitivity of the ORT in our study was $84.6 \%$ because of the small number of truepositive results confirmed by EIA, not because of reader error. Reader error may be reduced by providing training by experienced staff at the point of sale or point of testing.
Early infection: The ORT only detects HIV-1/2 antibody (which takes 2-4 weeks or more to develop), so early infections (eg, a positive result on an HIV p24 antigen test) may be missed. Such cases are viraemic and highly infectious, and identifying these is crucial to enable consideration of antiretroviral therapy and to prevent HIV disease progression and transmission.

Referring again to the study submitted to the US FDA Blood Products Advisory Committee, one of the eight participants reporting a nonreactive result was seroconverting. ${ }^{12}$ Similarly, in our study, we identified two asymptomatic MSM as having early infections. Together, the studies highlight the decreased sensitivity during early infection and the need to confirm non-reactive ORT results with serological tests - which underpins our decision to confirm all ORT results (reactive and non-reactive) with EIA.

For the general population at low risk of HIV infection, using serological tests to confirm reactive ORT results only (as recommended by the US FDA) is practical and appropriate. However, for those at high risk, like MSM, we suggest that clinicians consider confirmatory serological tests on a case-by-case basis. A careful history to identify recent exposure (eg, unprotected anal intercourse), symptoms of seroconversion (eg, flu-like illness, rash) and consideration of the "window period" (ie, time for HIV antibody to develop since the last potential exposure) will help inform this decision. In public NSW sexual health services, serological tests are routinely performed for syphilis and hepatitis $A, B$ and $C$, so requesting a 
confirmatory HIV antibody test is a simple matter.

The current recommendation for MSM is to have four HIV tests per year. ${ }^{10}$ Seventy per cent of the participants in our study did not follow this recommendation, and only $20 \%$ did. Ten per cent had never been tested for HIV. These findings suggest that more work is required to encourage MSM and individuals with numerous sexual partners or who engage in other high-risk behaviour to test quarterly for HIV.

Overall, participants were satisfied with the ORT, finding it less stressful than standard venous sampling. They would have future ORTs, recommend the test to peers and test more frequently if the TGA approved the ORT. Participants who usually had tests less frequently found the ORT less stressful than those who had quarterly tests. This was not because those who had more frequent tests reported more unsafe sex compared with those who had less frequent tests. It may simply be that those who had more frequent tests were more anxious and motivated to test often and to know their HIV serostatus.

\section{Conclusions}

With the incidence of HIV in Australia at its highest level since 1992, HIV rapid tests such as the ORT can play a key role in early diagnosis, initiation of antiretroviral therapy and preventing transmission, particularly among MSM and individuals at high risk. This will deliver clear individual and public health benefits.

We found that ORT specificity is very high for established infection but that its sensitivity is lower in early HIV infection. Thus, we suggest confirmatory serological tests for MSM and individuals at high risk or those with symptoms of seroconversion.

The ORT is a highly acceptable HIV screening test; our participants reported that they would recommend it to peers and, importantly, test more frequently if the test was approved by the TGA. Compared with the TGA-approved Alere Determine test, the ORT has similar sensitivity and specificity under both clinical trial conditions and in clinical use. However, the ORT has practical advantages that make it suitable for self-testing at home. As is the case in the US, we believe the ORT is an appropriate alternative rapid test to tackle Australia's HIV epidemic.

Acknowledgements: We thank the patients and staff at the Albion Centre, Kirketon Road Centre, Short Street Clinic, Western Sydney Sexual Health Centre and Taylor Square Private Clinic; Nicolas Parkhill and the AIDS Council of NSW; Katherine Brown and the Illawarra Sexual Health Service; and Mark Bloch and Holdsworth House Medical Practice. Also, we thank Colette McGrath and the South Eastern Sydney Local Health District HIV and Related Programs Unit for supporting the study and funding the test kits; Gilead Sciences Pty Ltd for funding the electronic patient registration kiosks in the Albion Centre; Quoc Pham and OraSure Technologies Inc for technical support; Ros Roots and Integrated Sciences Pty Ltd for training and supporting site investigators; and Helen Fraser and the South Eastern Sydney Local Health District Research Support Office.

Competing interests: No relevant disclosures.

Received 11 Sep 2014, accepted 13 Feb 2015.

References are available online at www.mja.com.au. 
1 The Kirby Institute. HIV, viral hepatitis and sexually transmissible infections in Australia. Annual surveillance report 2014. Sydney: The Kirby Institute, 2014. https://kirby.unsw.edu.au/sites/ default/files/hiv/resources/ASR2014. pdf (accessed Feb 2015).

2 Australian Government Department of Health. Seventh national HIV strategy 2014-2017. Canberra: DoH, 2014. http:// www.health.gov.au/internet/main/ publishing.nsf/Content/8E87E65EE F535B02CA257BF0001A4EB6/\$File/ HIV-Strategy2014-v3.pdf (accessed Feb 2015).

3 New South Wales Ministry of Health. HIV strategy 2012-2015. Quarter 32014 data report. Sydney: NSW Health. http://www.health.nsw.gov.au/ endinghiv/Documents/hiv-in-nsw3rd-quarter-report-2014.pdf (accessed Feb 2015).

4 New South Wales Ministry of Health. NSW HIV Strategy 2012
--2015; a new era. Sydney: NSW Health, 2012. http://www.health.nsw. gov.au/publications/Publications/ nsw-hiv-strategy-2012-15.pdf (accessed Feb 2015).

5 Kellerman SE, Lehman JS, Lansky A, et al. HIV testing within at-risk populations in the United States and the reasons for seeking or avoiding HIV testing. JAIDS 2002; 31: 202-210.

6 Lorenc T, Marrero-Guillamon I, Llewellyn A, et al. HIV testing among men who have sex with men (MSM): systematic review of qualitative evidence. Health Educ Res 2011; 26: 834-846.

7 Young SD, Bendavid E. The relationship between HIV testing, stigma, and health. AIDS Care 2010; 22: 373-380.

8 Inverness Medical. Determine HIV-1/2 Ag/Ab Combo Test package insert. Tokyo: Inverness Medical, 2009.
9 OraSure Technologies Inc. OraQuick ADVANCE Rapid HIV-1/2 Antibody Test package insert. Pennsylvania: OraSure Technologies, 2010.

10 Templeton D, Read P, Varma R, Bourne C. Australian sexually transmissible infection and HIV testing guidelines for asymptomatic men who have sex with men 2014: a review of the evidence. Sex Health 2014; 11: 217-229.

1 Conway DP, Holt M, McNulty A, et al. Multi-centre evaluation of the Determine HIV Combo assay when used for point of care testing in a high risk clinic-based population. PLOS One 2014; 8: e94062. http://journals.plos. org/plosone/article?id=10.1371/journal. pone.0094062 (accessed Dec 2014).

12 OraSure Technologies. OraQuick In-Home HIV Test. Blood Products Advisory Committee briefing document. Pennsylvania: OraSure Technologies, 2012. 